\title{
Ultrasound Biomicroscopic Evaluation of Anterior Segment Parameters and Surgical Area Following Trabeculectomy Surgery
}

\author{
Cem Kesim, ${ }^{1}$ (D) Nese Alagoz, ${ }^{2}$ (D) Banu Solmaz, ${ }^{2}$ () Cigdem Altan, $^{2}$ (i) Berna Basarir, ${ }^{2}$ (b) Muhittin Taskapili ${ }^{2}$ \\ ${ }^{1}$ Department of Ophthalmology, Koc University Faculty of Medicine, Istanbul, Turkey \\ ${ }^{2}$ Department of Ophthalmology, Beyoglu Eye Training and Research Hospital, Istanbul, Turkey
}

\begin{abstract}
Objectives: This study used ultrasound biomicroscopy (UBM) imaging to evaluate the anterior segment structure and surgical area of patients with open angle glaucoma following a trabeculectomy procedure.

Methods: Consecutive patients with primary open angle or pseudoexfoliative glaucoma who underwent a trabeculectomy were included. UBM was used to examine the anatomical parameters of anterior chamber depth, anterior chamber angle, angle opening distance at $500 \mu \mathrm{m}$ (AOD500), trabecular ciliary process distance (TCPD), iris ciliary process distance, scleral ciliary process angle (SCPA), and ciliary process thickness, as well as the internal ostium opening, ostium-iris distance, and scleral flap dimensions. Bleb morphologies were evaluated qualitatively using the Yamamoto classification and quantitatively according to the presence of drainage from the medial, lateral, and posterior borders.

Results: Twenty-five eyes of 23 patients ( 4 female, 19 male) were included in the study. The TCPD and SCPA measurements were significantly greater in the postoperative third month $\left(1.04 \pm 0.12 \mathrm{~mm}\right.$ vs $1.09 \pm 0.16 \mathrm{~mm}, \mathrm{p}<0.05 ; 58.6 \pm 3.9^{\circ}$ vs $60.8 \pm 3.8^{\circ}, p<0.05$, respectively). Cases with a longer ostium-iris distance had a significantly greater AOD500, TCPD, and SCPA $(p<0.05)$. Bleb drainage and morphological classification were correlated $(p=0.00 \mathrm{l})$.

Conclusion: UBM is a sound and efficient method to investigate anterior segment variations and the surgical bleb following a trabeculectomy. TCPD and SCPA values increased after surgery.
\end{abstract}

Keywords: Anterior segment, filtering bleb, open angle glaucoma, trabeculectomy, ultrasound biomicroscopy.

\section{Introduction}

Trabeculectomy is the principal surgical treatment method to reduce intraocular pressure (IOP) in glaucoma patients (I). The main objective in this procedure is to create a filtering bleb between the anterior chamber and the subconjunctival space in order to obtain controlled excessive aqueous fluid drainage via the transconjunctival and episcleral venous pathways (2). Although a surgical success rate of up to $90 \%$ has been reported with this procedure, (I) various complications related to the bleb (fibrosis, early or late leakage) are frequently encountered $(3,4)$. Various efforts to overcome fibrosis-related bleb failure through preoperative and postoperative interventions have been introduced, such as the use of antimetabolites like mitomycin-C intraoperatively (5) and bleb needling on short term follow-up (6).

Ultrasound biomicroscopy (UBM) was first developed as a novel imaging technique by Pavlin et al. (7) in 1991. Highfrequency $(35-50 \mathrm{MHz})$ ultrasound waves are used to enhance high-resolution anterior segment imaging with sufficient depth to visualize ciliary processes. Predefined length 
and angle measurements (8) made UBM a crucial tool to perform qualitative and quantitative analyses in various pathologies and treatments related to glaucomatous diseases.

In addition to reducing IOP through the fistula created between the anterior chamber and the subconjunctival space, trabeculectomy surgery can also have an impact on the anterior segment anatomy of the eye. The objective of this study was to use UBM imaging to examine anatomical anterior segment changes in eyes treated with trabeculectomy surgery. The surgical bleb morphology was analyzed and aqueous drainage routes were assessed in order to evaluate bleb functioning with quantitative data.

\section{Methods}

Patients who underwent trabeculectomy surgery with mitomycin $C$ between June 2016 and February 2017 at Beyoglu Eye Training and Research Hospital were included in this consecutive, retrospective study. All patients with preoperative and postoperative 3-month anterior segment UBM imaging data were included. Patients with previous glaucoma surgery or with optic nerve disease other than glaucoma were excluded. Written, informed consent was provided by each patient. All of the procedures performed conformed with the tenets of the Declaration of Helsinki. This study was approved by the local Institutional Ethics Committee.

Trabeculectomy surgeries were performed using fornixbased flaps and no peroperative complications occurred during surgery. The target of success for IOP reduction was an IOP of between $5 \mathrm{mmHg}$ and $18 \mathrm{mmHg}$. Cases within this range without any antiglaucomatous agent were considered a complete success, and cases remaining within this range with medication were accepted as partially successful. Cases with a result that remained below $5 \mathrm{mmHg}$ or above $18 \mathrm{mmHg}$ in any condition were considered surgical failures.

UBM examinations were performed using the VuMax II ophthalmic ultrasound system with a $35 \mathrm{MHz}$ transducer (Sonomed Escalon Inc., Lake Success, NY, USA). Imaging procedures were performed in dim light conditions with the patient in the supine position. Scanning examination of the anterior segment angle and the filtering bleb was performed with an over-sized immersion cup and $10 \mathrm{~mL}$ isotonic $0.9 \%$ sodium chloride solution as the immersion liquid. Four horizontal and vertical quadrants were used to evaluate anterior chamber depth (ACD) and the anterior chamber angle (ACA) in a central anteroposterior scan, the angle opening distance to the scleral spur at $500 \mu \mathrm{m}$ (AOD500), and the trabecular ciliary process distance (TCPD), the iris ciliary process distance (ICPD), the scleral ciliary process angle (SCPA), and the ciliary process thickness in a radial scan (Fig. I). The newly introduced internal ostium opening, ostium-iris distance, and scleral flap dimensions in the area of surgery were assessed with radial and transverse scans (Fig. 2) using a built-in caliper and calculated by the device software program. The cases were divided into 2 subgroups according to a preoperative ACA width of $40^{\circ}$. Qualitative bleb morphology was evaluated according to the Yamamoto classification, (9) and bleb drainage tracts in the lateral, medial, and posterior borders were noted for further quantitative investigation. In this assessment, a continuing hypoechogeneity between the scleral flap route and the bleb cavity for each flap border was considered a patent drainage route (Fig. 3).

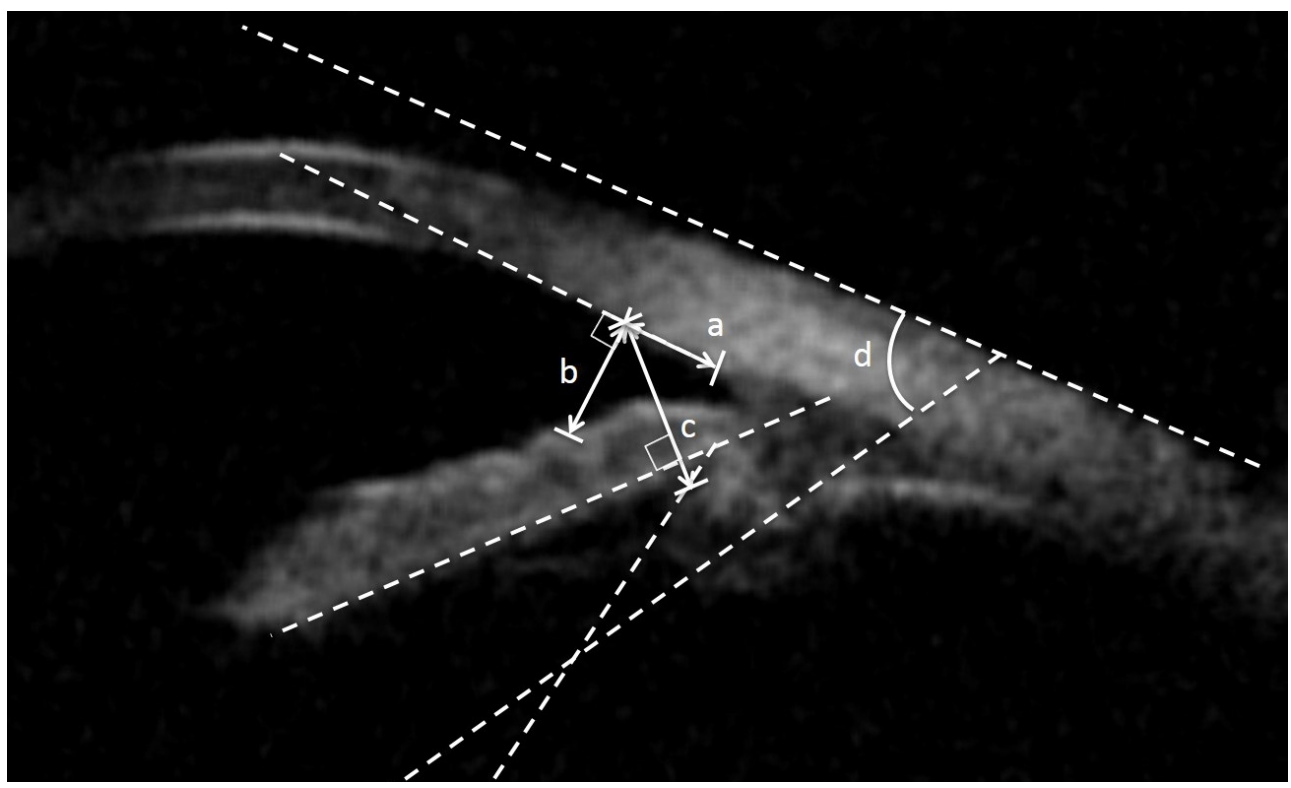

Figure I. Anterior segment parameter measurements. a) Measurement of $500 \mu \mathrm{m}$ from the scleral spur, b) Angle opening distance at $500 \mu \mathrm{m}, \mathrm{c}$ ) Trabecular ciliary process distance, d) Scleral ciliary process angle. 


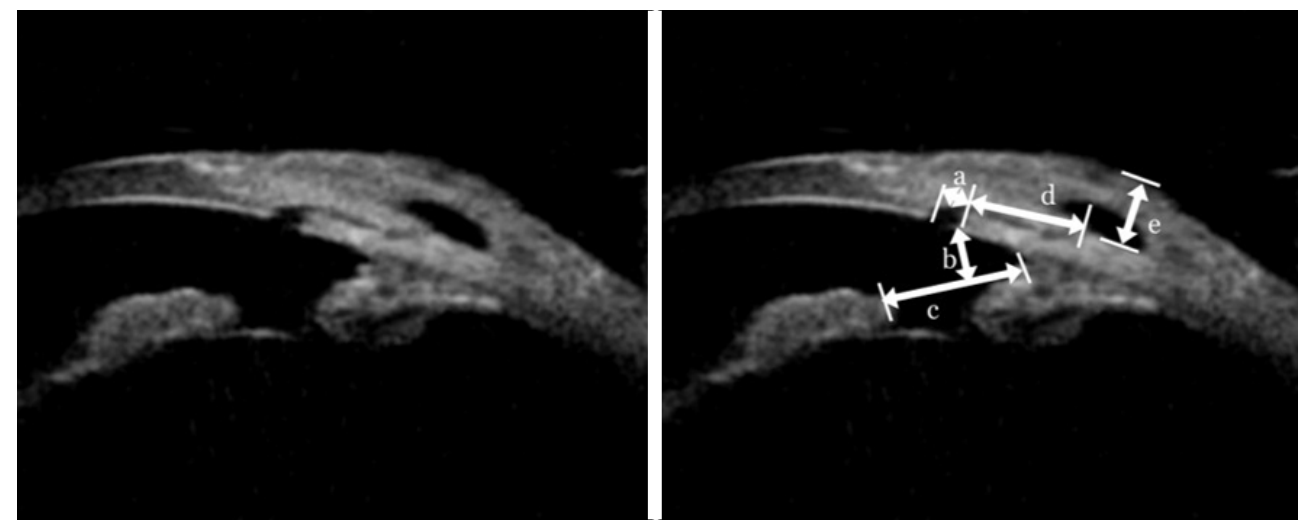

Figure 2. Ultrasound biomicroscopy view of the surgical area. Left: A visible hypoechogenic route between the ostium and bleb cavity shows an open posterior draining border. Right: Surgical distance measurements are illustrated with two-headed arrows. Legend: a) Internal ostium, b) Ostium-iris distance, c) Peripherical iridectomy opening, d) Scleral flap antero-posterior width, e) Bleb height.

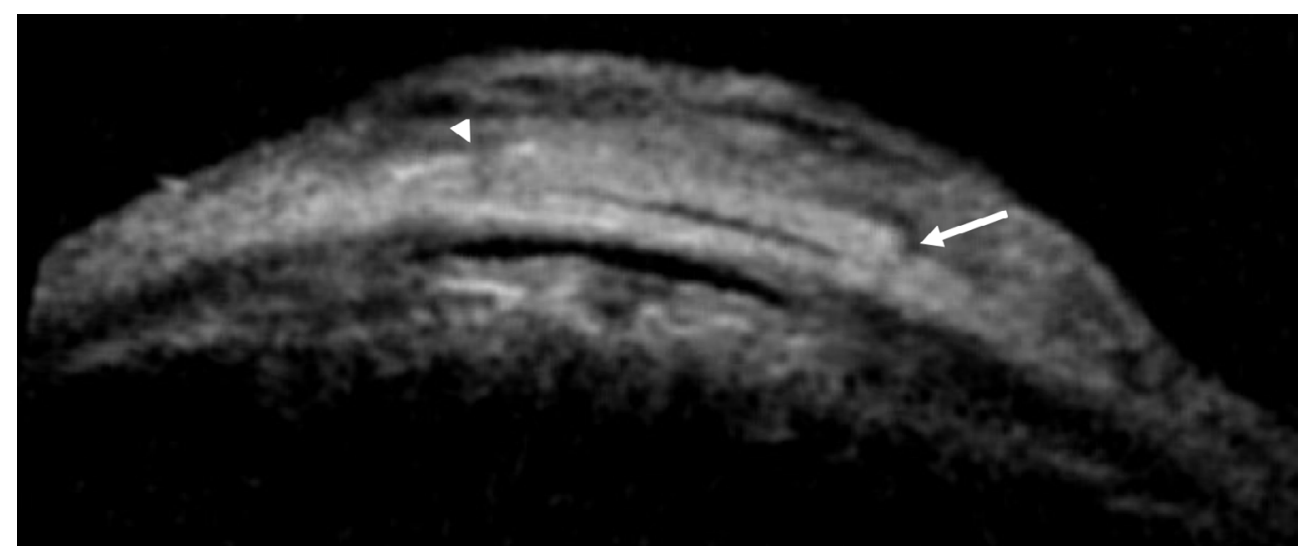

Figure 3. Transverse view of the surgical area at the postoperative third month. The right side of the image shows the lateral side of the eye.A patent lateral draining border (arrow) and an occluded medial border (arrowhead) are visible.

The statistical analysis was performed with IBM SPSS Statistics for Windows, Version 22.0 (IBM Corp., Armonk, NY, USA) Data were expressed as mean \pm SD for metric values and frequency was calculated as a percentage for categorical variables. The Wilcoxon signed rank and Mann-Whitney $U$ tests were performed for dependent and independent values, respectively. Analysis between categorical values was performed with a chi-square test. Spearman correlation coefficient $\rho$ values were used to investigate the association between different variables.

\section{Results}

Twenty-five eyes of 23 patients ( 19 male and 4 female) were included in this study. The mean age was $61.9 \pm 9.84$ years. Of 25 eyes, $13(52 \%)$ had primary open angle glaucoma and 12 (48\%) had pseudoexfoliative glaucoma. The mean baseline best corrected visual acuity (BCVA) was $0.83 \pm 0.86$ using $\log M A R$ scoring, and the eyes had a mean baseline IOP of $24.5 \pm 6.57 \mathrm{mmHg}$ measured with Goldmann applanation tonometry, with a mean of $3.6 \pm 0.9 \mathrm{mmHg}$ in those using antiglaucoma medication. All but I patient was phakic. At the third month, the mean BCVA remained $0.84 \pm 0.62$, and there was a significant reduction in the mean IOP, with a mean II.I $\pm 4.4 \mathrm{mmHg}$ (mean with antiglaucoma medication: $0.61 \pm 0.98 \mathrm{mmHg})$. Thirteen $(52 \%)$ eyes had complete surgical success, while 10 eyes (40\%) had partial success and 2 eyes $(8 \%)$ were considered a failure. No significant difference was detected in the results between the POAG and PEXG groups in terms of surgical success or anterior segment findings, surgical area, or bleb morphology following surgery.

Anterior segment findings: The UBM findings of anterior segmental changes were documented as shown in Table I: At the third month, the ACD, AOD500, ACA, and ICPD measurements demonstrated no significant change, whereas the TCPD and SCPA measurements had significantly increased (TCPD: $1.04 \pm 0.12 \mathrm{~mm}$ vs $1.09 \pm 0.16 \mathrm{~mm}$, $p=0.044$; SCPA: $58.6 \pm 3.9^{\circ}$ vs $60.8 \pm 3.8^{\circ} ; p=0.027$ ). Significantly increased AOD500 and SCPA measurements were found in the narrow angle case subgroup where the preoperative ACA was $<40^{\circ}$ ( $p=0.028$ both), while no signifi- 
Table I. Changes in anterior segment parameters at the postoperative third month

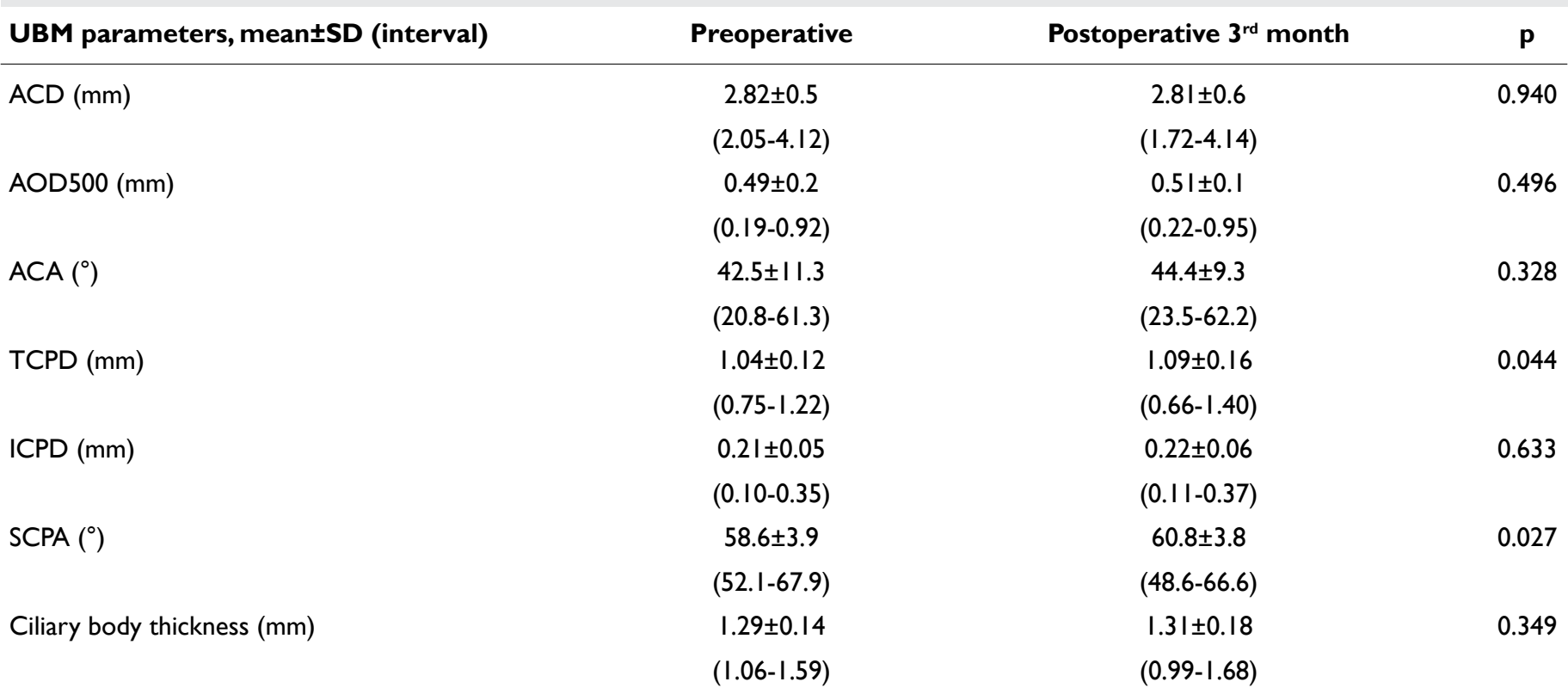

ACA:Anterior chamber angle;ACD:Anterior chamber depth;AOD500:Angle opening distance at 500 um; ICPD: Iris ciliary process distance; SCPA: Scleral ciliary process angle;TCPD:Trabecular ciliary process distance; UBM: Ultrasound biomicroscopy.

cant change was noted for any other parameter in the case subgroup where the ACA was $>40^{\circ}$. There was a negative correlation between preoperative AOD500 values and the change in the postoperative third month $(p=-0.526$, $p=0.007)$ as well as between the preoperative SCPA values and the change in the postoperative third month $(p=$ $0.617, p=0.001$ ).

Filtering bleb findings: The filtering blebs were classified quantitatively according to patency in the lateral, medial, and posterior borders. Blebs with drainage at I border were included in Group I, and blebs with drainage at 2 or 3 borders in Group II. The qualitative classification was performed according to the categories described by Yamamoto (9). Blebs classified as type $L$ (low-reflective) were included in Group A, and type $H$ (high-reflective), type $E$ (encapsulated), and type $F$ (flattened) were included in Group B.
Categorical analysis between Groups I-II and Groups A-B revealed a statistically significant compatibility between bleb drainage and bleb echogeneity $(p=0.001)$. Bleb drainage, echogeneity, and surgical success outcomes are presented in Table 2. One patient was determined to have iris incarceration in the internal ostium and underwent surgical revision. The patient had partial success with medically controlled IOP following surgery.

Surgical area findings: The findings related to surgical area measurements are given in Table 3. Among the new surgical parameters introduced in our study, the variation in internal ostium width and peripheral iridectomy width, as well as scleral flap dimensions (antero-posterior and temporo-nasal lengths with flap thickness) were found to have no correlation with surgery results. On the other hand, ostium-iris distance measurements were associated with sev-

Table 2. Filtering bleb drainage, echogeneity, and surgical success outcomes at the third month

\begin{tabular}{|c|c|c|c|c|c|c|c|c|}
\hline \multirow[t]{2}{*}{ Bleb drainage, $n,(\%)$} & \multirow[t]{2}{*}{$\begin{array}{c}\text { Bleb height, } \\
\text { mean } \pm S D(m m)\end{array}$} & \multicolumn{4}{|c|}{$\begin{array}{l}\text { Bleb echogeneity } \\
\text { (Yamamoto) n, (\%) }\end{array}$} & \multicolumn{3}{|c|}{$\begin{array}{c}\text { Surgical success } \\
\text { n, (\%) }\end{array}$} \\
\hline & & $\mathbf{L}$ & $\mathbf{H}$ & $\mathbf{E}$ & $\mathbf{F}$ & Full & Partial & Failure \\
\hline $8(32)$ & $1.26 \pm 0.56$ & $2(8)$ & $2(8)$ & $2(8)$ & $2(8)$ & $3(12)$ & $3(12)$ & $2(8)$ \\
\hline $10(40)$ & $1.29 \pm 0.35$ & $9(36)$ & I (4) & 0 & 0 & $5(20)$ & $5(20)$ & 0 \\
\hline $7(28)$ & $1.54 \pm 0.22$ & $7(28)$ & 0 & 0 & 0 & $5(20)$ & $2(8)$ & 0 \\
\hline
\end{tabular}

E: Encapsulated; F: Flat; H: High echogeneity; L: Low echogeneity. 
Table 3. Surgical area findings at the third month

Surgical area parameters $(\mathrm{mm})$

Mean $\pm S D$ (interval)

Postoperative

Internal ostium width

$3^{\text {rd }}$ month

$0.59 \pm 0.20$

(0.14-1.08)

Ostium-iris distance

$0.45 \pm 0.19$

(0.06-0.79)

Peripherical iridectomy width (antero-posterior)

$1.45 \pm 0.38$

(0.74-2.22)

Scleral flap width (antero-posterior)

$1.88 \pm 0.42$

$(1.06-2.80)$

Scleral flap width (temporo-nasal)

$4.01 \pm 0.53$

(3.04-5.30)

$0.44 \pm 0.09$

Scleral flap thickness

$(0.25-0.64)$

Bleb height

$1.35 \pm 0.42$

$(0.44-2.13)$

eral anterior segment changes. When the cases were divided into 2 subgroups based on an ostium-iris distance of 0.44 $\mathrm{mm}$, cases with a highly positioned internal ostia $(>0.44 \mathrm{~mm})$ were found to have significantly increased AOD500, TCPD, and SCPA measurements $(p=0.045,0.005$, and 0.034 respectively). The findings also demonstrated a weakly positive correlation for AOD500 and TCPD $(p=0.402, p=0.047$ and $p=0.466, p=0.019$ ).

Correlation analysis of surgical parameters revealed a weakly negative correlation between ostium width (a), scleral flap thickness (b), and bleb height (c) [between (a) and $(c): p=-0.408, p=0.043$; between $(b)$ and $(c): p=-0.464$, $p=0.020]$.

\section{Discussion}

Trabeculectomy is still considered the gold standard surgical treatment for medically intractable glaucoma. However, many complications may arise, such as bleb hypo/ hyperfunctioning, anterior chamber narrowing, and bleb leakage (3); therefore, proper postoperative control of the anterior chamber and surgical bleb area is critical. Anterior segment imaging techniques, such as anterior segment ocular computed tomography (AS-OCT) and UBM have become very useful tools to make these assessments and provide further information about the anterior segment anatomy (I0).

\section{Bleb Morphology}

Yamamoto et al. (9) introduced the primary research of bleb morphology and its qualitative analysis in a II7-eye study in which surgical blebs were evaluated according to the I) presence of a scleral route, 2) internal bleb reflectivity, and 3 ) intra-bleb cystic formations, and the blebs were then classified in 4 groups:

L: low-reflective

$\mathrm{H}$ : high-reflective

E: encapsulated

F: flat

In that study, among 82 eyes with surgical success which required no postoperative additional antiglaucomatous medication, 79 (96\%) were found to be L-type blebs. The prediction that hyporeflective blebs have a good prognosis was endorsed by AS-OCT imaging performed of bleb walls by Nakano et al. (II), which revealed intramural microcystic aqueous pouches in the bleb and related this finding with impaired bleb fibrosis and resulting greater success. Other studies conducted by McWhae (12) and Avitabile (13), in which surgical blebs were classified in terms of internal reflectivity and scleral route potency as good, medium, or weak, have shown strong correlations between the UBM classification and postoperative IOP control. In our study, we had 12 eyes with an L-type bleb among 13 surgically successful eyes (92\%). Our quantitative analysis based upon a bleb drainage count revealed that 2 L-type, 2 H-type, 2 Etype, and 2 F-type blebs had a single draining border; 9 Ltype and I $\mathrm{H}$-type blebs had 2 draining borders; and that all 7 blebs with 3 draining borders were L-type blebs. A similar study reported by El Salhy et al. (14) scored filtering blebs according to 4 categorical UBM findings: intrableb reflectivity, visibility of subscleral tract, bleb height, and the presence of bleb cysts. They observed a greater success rate among patients with higher score. While they investigated the patency of the subscleral tract in the radial plane, in our study, we additionally evaluated the transverse plane in order to evaluate the lateral and medial borders in order to improve our quantitative analysis. Our statistical analysis also confirmed that our quantitative drainage analysis was in correlation with the Yamamoto classification.

\section{Surgical Area}

Our study findings suggest that surgical preferences for scleral flap dimensions and internal ostium width and height may not affect the endpoint surgical result of a lower IOP. In addition, possible impacts to anterior segment anatomy deserve consideration. The effect of an internal ostium location on the AOD500, TCPD, or SCPA may have implications that are described below; however, these findings should be confirmed with further investigation and the mechanisms remain to be elucidated.

\section{Changes in Anterior Segment Anatomy}

Few studies could be found in the literature about anterior 
segment changes following trabeculectomy other than those evaluating bleb morphology. In one of these studies, MartinezBello et al. ( 15 ) evaluated anterior segment changes in single trabeculectomy $(n=17)$ and combined trabeculectomy+cataract surgery $(n=13)$ procedures in POAG and PEXG patients. An increase in AOD500 measurements was found in both groups, as well as an ACD increase in the combined group. Park et al. (16) conducted a more detailed study, which examined a 56-eye population diagnosed with primary angle closure glaucoma (PACG): 26 eyes underwent single trabeculectomy and 29 eyes underwent a triple procedure. Significant increases in the ACD, AOD500, TCPD, trabecular iris angle, SCPA, scleral iris angle, and angle recess area parameters were found in the triple-procedure group, while the single-surgery group had only ACD, AOD500, and ARA increases.

Our study consisted of cases with a similar background to those of the Martinez-Bello study (I3 POAG and 12 PEXG cases). Unlike other studies, we detected significant increases in SCPA and TCPD measurements in our open angled cases following a simple trabeculectomy. However, cases with these significant increases were in the subgroup with a lower initial ACA. An additional increase in AOD500 for this subgroup corresponds with the results in Park's study. These findings are strengthened with correlation analyses revealing that cases with narrower baseline AOD500 and SCPA values demonstrated more significant increases postoperatively. However, it should be noted that these previous studies included combined surgeries, which makes it problematic to compare these studies directly with our uniform trabeculectomy surgery findings. Increases in the AOD500, TCPD, and SCPA occurring at the same time might imply a posterior rotation of the ciliary body in susceptible cases following a single trabeculectomy. The increase in SCPA and TCPD following trabeculectomy raises the question of whether a single trabeculectomy also results in posterior rotation of ciliary body in cases with plateau iris configuration. Further studies that include cases with a narrow angle and plateau iris configuration could provide valuable detailed results.

\section{Limitations}

The limitations of our study are based on several factors. Compared with the large study population of Yamamoto, our 25-eye study might have had different outcomes if a larger number of patients had been enrolled. The similar case groups of Martinez-Bello and Park enabled a comparison of the results. Another limiting factor is that all of the UBM images were assessed by a single author (C.K.), which means the study does not have an interobserver reliability test $(17,18)$. Nevertheless, high intraobserver reliability is attainable with UBM imaging, (I7) which makes deductions made using UBM in clinical practice acceptable measures. The absence of PACG and plateau iris configuration cases is also a limiting factor.

\section{Conclusion}

Trabeculectomy surgery has an impact on the anterior segment anatomy that can be detected using UBM imaging. The increase in TCPD and SCPA measurements that we found in our study suggested a posteriorly inclined ciliary body on its radial axis following surgery. The positioning of the internal ostium might also affect these changes. Further investigations to clarify and confirm the mechanisms of these findings should be conducted. Moreover, quantitative analysis to detect bleb drainage around the borders might provide beneficial clinical findings to predict surgical success.

\section{Disclosures}

Ethics Committee Approval: The Ethics Committee of Istanbul Bagcilar Training and Research Hospital provided the ethics committee approval for this study (13.06.2017-2017-578).

Peer-review: Externally peer-reviewed.

Conflict of Interest: None declared.

Authorship Contributions: Involved in design and conduct of the study (CK, NA, BS); preparation and review of the study (CK, NA, CA); data collection (CK, BS); and statistical analysis (CK, NA).

\section{References}

I. Grehn F, Hollo G, Lachtar Y, Migdal C, Thygesen J. Incisional Surgery, Treatment Principles and Options. In: Traverso CE, editor. Terminology and Guidelines for Glaucoma. 4th ed. Savona, Italy: PubliComm; 20I4. p. 169-7I.

2. Razeghinejad MR, Fudemberg SJ, Spaeth GL. The changing conceptual basis of trabeculectomy: a review of past and current surgical techniques. Surv Ophthalmol 2012;57:I-25.[CrossRef]

3. Liebmann LM, Ritch R. Complications of glaucoma filtering surgery. In: Ritch R, Shields B, Krupin T, editors. The Glaucomas. St. Louis: CV Mosby; 1996. p. 1703-36.

4. Herndon LW. Complications of Glaucoma Surgery and their Management. In: Yanoff M, Duker JS, editors. Ophthalmology. 4th ed. Elsevier Saunders; 2013.

5. Chen CW, Huang HT, Bair JS, Lee CC. Trabeculectomy with simultaneous topical application of mitomycin-C in refractory glaucoma. J Ocul Pharmacol 1990;6: 175-82. [CrossRef]

6. Greenfield DS, Miller MP, Suner IJ, Palmberg PF. Needle elevation of the scleral flap for failing filtration blebs after trabeculectomy with mitomycin-C. Am J Ophthalmol 1996; 1 22: 195-204.

7. Pavlin CJ, Harasiewicz K, Sherar MD, Foster FS. Clinical use of ultrasound biomicroscopy. Ophthalmology 1991;98:287-95.

8. Pavlin CJ, Harasiewicz K, Foster FS. Ultrasound biomicroscopy of anterior segment structures in normal and glaucomatous eyes. Am J Ophthalmol 1992; I 13:38I-9. [CrossRef]

9. Yamamoto T, Sakuma T, Kitazawa Y. An ultrasound biomicroscopic study of filtering blebs after mitomycin $C$ trabeculectomy. Ophthalmology 1995; 102:1770-6. [CrossRef]

10. Golez E 3rd, Latina M. The use of anterior segment imaging after 
trabeculectomy. Semin Ophthalmol 2012;27:155-9. [CrossRef]

I I. Nakano N, Hangai M, Nakanishi H, Inoue R, Unoki N, Hirose F, et al. Early trabeculectomy bleb walls on anterior-segment optical coherence tomography. Graefes Arch Clin Exp Ophthalmol 2010;248: I I73-82. [CrossRef]

12. McWhae JA, Crichton AC. The use of ultrasound biomicroscopy following trabeculectomy. Can J Ophthalmol 1996;31:187-91.

13. Avitabile T, Russo V, Uva MG, Marino A, Castiglione F, Reibaldi A. Ultrasound- biomicroscopic evaluation of filtering blebs after laser suture lysis trabeculectomy. Ophthalmologica 1998;2 | 2:|7-2|. [CrossRef]

14. El Salhy AA, Elseht RM, AI Maria AF, Shalaby SMAE, Hossein TR. Functional evaluation of the filtering bleb by ultrasound biomicroscopy after trabeculectomy with mitomycin C. Int J Ophthalmol 2018; I I:245-50.
15. Martínez-Belló C, Rodriguez-Ares T, Pazos B, Capeáns C, Sánchez-Salorio M. Changes in anterior chamber depth and angle width after filtration surgery: A quantitative study using ultrasound biomicroscopy. J Glaucoma 2000;9:5 I-5. [CrossRef]

16. Park SW, Heo H, Yang KJ. Comparison of ultrasound biomicroscopic changes after glaucoma triple procedure and trabeculectomy in eyes with primary angle closure glaucoma. J Glaucoma 2009; | 8:3 I I-5.[CrossRef]

17. Tello C, Liebmann J, Potash SD, Cohen H, Ritch R. Measurement of ultrasound biomicroscopy images: intraobserver and interobserver reliability. Invest Ophthalmol Vis Sci 1994;35:3549-52.

18. Marchini G, Pagliarusco A, Toscano A, Tosi R, Brunelli C, Bonomi L. Ultrasound biomicroscopic and conventional ultrasonographic study of ocular dimensions in primary angle closure glaucoma. Ophthalmology 1998;|05:209|-8. [CrossRef] 